\title{
MORTALITY RATE OF ICU PATIENTS WITH \\ THE MIDDLE EAST RESPIRATORY SYNDROME \\ - CORONAVIRUS INFECTION AT KING FAHAD HOSPITAL, JEDDAH, SAUDI ARABIA
}

\author{
Mohammed A. Garout ${ }^{1}$, Hani A.A. Jokhdar ${ }^{1}$, Imad A. Aljahdali', Ahmed R. Zein ${ }^{2}$, Reda A. Goweda ${ }^{1,3}$, Abdurahman \\ Hassan-Hussein ${ }^{1}$ \\ 'Department of Community Medicine, Faculty of Medicine, Umm Al-Qura University, Makkah, Kingdom of Saudi Arabia \\ ${ }^{2}$ Intensive Care Department, King Fahad Hospital, Jeddah, Kingdom of Saudi Arabia \\ ${ }^{3}$ Department of Family Medicine, Faculty of Medicine, Suez Canal University, Egypt
}

\section{SUMMARY}

Objective: The Middle East Respiratory Syndrome Coronavirus (MERS-CoV) is a novel coronavirus circulating in the Arabian Peninsula since September 2012. It leads to significant respiratory disease and among patients with co-morbidities is associated with high mortality. This research studied the mortality rate of MERS-CoV among intensive care unit (ICU) patients and the correlation of mortality with different co-morbidities.

Methods: This was a retrospective observational study conducted at the Intensive Care Unit of the King Fahad Hospital, Jeddah, Saudi Arabia. Data was obtained through patient chart review.

Results: The total sample consisted of 52 laboratory-confirmed MERS-CoV infection patients. 39 patients died, with a $75 \%$ case-fatality rate. Many patients had underlying co-morbidities, including diabetes mellitus (51.9\%), hypertension (46.2\%), and chronic renal disease (21.2\%).

Conclusions: MERS-CoV ICU mortality remains markedly high due to a combination of factors; the disease process of MERS-CoV leads to multiple organ failure, particularly respiratory and renal failure.

Key words: Middle East Respiratory Syndrome Coronavirus, mortality, intensive care units, Saudi Arabia

Address for correspondence: R. Goweda, Department of Family Medicine, Suez Canal University. Kilo 4.5, 41522 Ismailia, Egypt. E-mail: redagoweda@yahoo.com

https://doi.org/10.21101/cejph.a4764

\section{INTRODUCTION}

A novel coronavirus leading to significant respiratory disease, termed the Middle East Respiratory Syndrome - Coronavirus (MERS-CoV), has been circulating in the Arabian Peninsula since September 2012. As of December 2014, 781 cases were reported in the Kingdom of Saudi Arabia (KSA), representing $86 \%$ of worldwide infections; overall $95 \%$ of cases originated in the nations of the Arabian Peninsula (KSA, United Arab Emirates (UAE), Qatar, Kuwait, Yemen, and Oman). Approximately $40 \%$ of patients diagnosed with MERS-CoV infection have died (1). As of December 2014, two travel-associated cases were diagnosed in the United States in healthcare workers originating from Saudi Arabia (2).

MERS-CoV is a SARS-like virus presenting with flu-like symptoms. In a 2013 review of 47 laboratory-confirmed cases in Saudi Arabia, patients typically presented with symptoms of fever in $98 \%$ of cases, cough in $83 \%$ of cases, shortness of breath in $72 \%$ of cases, and myalgia in $32 \%$ of cases. Gastrointestinal symptoms were experienced less often with diarrhea in $26 \%$ of cases, vomiting in $21 \%$ of cases, and abdominal pain in $17 \%$ of cases. $72 \%$ of patients required mechanical ventilation. Most patients were male, with a ratio of 3.3 males for every female (3).

MERS-CoV has been reported in a wide spectrum from the asymptomatic patient to severe pneumonia resulting in an acute respiratory distress syndrome (ARDS) (3). Disseminated intravascular coagulation (DIC), pericarditis, and in particular renal failure were often seen (4).

The mode of transmission of MERS-CoV was shown to be via close contact, but without a persistent community transmission (5). Transmission in KSA occurred predominantly via person-toperson contact with infected persons, particularly from patients to healthcare staff during nosocomial outbreaks at several hospitals (6). Also at-risk were immediate contacts such as family, friends, caregivers and other close contacts of people with suspected or confirmed MERS-CoV infection (6).

The World Health Organization (WHO) website on coronavirus infections distributes technical guidelines on MERS-CoV surveillance and investigation, infection prevention and control, laboratory testing, as well as case definitions. The most current interim case definition allows for identification of a confirmed case in individuals with a positive lab test by molecular diagnostics including either a positive polymerase chain reaction (PCR) 
on at least two specific genomic targets or a single positive target with sequencing on a second (7).

Chest radiographs typically demonstrate bilateral patchy infiltrates consistent with viral pneumonitis and Acute Respiratory Distress Syndrome (ARDS). The lower lobes are more often affected. CT scans demonstrate interstitial infiltrates. Laboratory findings show thrombocytopenia and leucopenia, in particular lymphopenia (8).

Patient samples containing the highest viral loads for diagnostic PCR testing can be obtained from the lower respiratory tract via sputum sample, tracheal aspirate, or bronchoalveolar lavage (9). Upper respiratory tract sampling via nasopharyngeal swab is less invasive and in practice acceptable for diagnosis (3).

After a patient exhibits symptoms, the United States Centers for Disease Control and Prevention (CDC) recommends collecting several specimens from different sites at various time points. Strict infection-control measures should be observed during collection. Protocols guide to the appropriate personal protective equipment, methods of collection, as well as to specimen handling, storage, and shipping or transfer to approved laboratories (10).

Rapid and diagnostic MERS-CoV case confirmation is achieved with several highly sensitive, real-time RT-PCR assays (11).

Potential treatment of the novel coronavirus is hoped for with suppressive immunotherapies. Kindler et al. demonstrated in vitro treatment with exogenous interferon (IFN), particularly IFN- $\alpha$ and IFN- $\lambda$, effectively reduced viral replication, overcoming MERS$\mathrm{CoV}$ tendency to antagonize endogenous IFN production (12). A study by Falzarano et al. describes rhesus macaques which were given interferon- $\alpha 2 b$ and ribavirin and exposed to MERS-CoV; they subsequently developed less pneumonia than control animals (13). A Saudi observational study of five critically ill patients with MERS-CoV with ARDS and on ventilators were given a trial of interferon- $\alpha 2 b$ and ribavirin; however, all succumbed to the disease. Very late initiation of antiviral treatment was thought to be an influencing factor, and it remains to be seen whether it may have benefit earlier in the course of disease (14). Ren et al. have proposed a potential therapy of viral protease inhibition (15).

MERS-CoV currently has no vaccine and no specific treatment is recommended, management remains supportive care (6). Among the most specialized support patients receive is extracorporeal membrane oxygenation (ECMO), a life-saving ICU mechanism for markedly distressed patients, with inadequate cardiac and/or respiratory func- tion. ECMO transiently and mechanically supplants cardiac and/or respiratory function. There are dedicated ECMO teams, consisting of ICU specialists, respiratory therapists and ECMO specialists, who continually monitor patients during ECMO treatment (16).

\section{MATERIALS AND METHODS}

This was a retrospective observational study conducted at the Intensive Care Unit (ICU) of the King Fahad Hospital, Jeddah (KFHJ), Kingdom of Saudi Arabia from March 2014 to July 2014. KFHJ became a near-dedicated MERS-CoV referral centre for Jeddah and the western third of Saudi Arabia during the fourmonth duration of the study. The MERS-CoV-dedicated ICU of KFHJ had a total of 18 beds. The study had a comprehensive sample of 52 ICU patients admitted for MERS-CoV.

Patient flow through KFHJ to the ICU consisted of two pathways: either direct admission of patients with proven MERS-CoV from other hospitals or through the KFHJ Emergency Department (ER) based on meeting the WHO MERS-CoV criteria. At the beginning of the study period, which corresponded with one of KSA's MERS-CoV outbreak peaks, KFHJ was segregated into two sections: a MERS-CoV-dedicated wing consisting of part of the ER and a separate and new tower building, and the main hospital building for all other patients not suspected to have MERS-CoV.

For patients admitted through the ER, a triage system was established to filter suspected cases. This filtration system consisted of ER specialists under supervision of ER consultants and infection control teams that applied the criteria developed by the Saudi Ministry of Health (MOH) Scientific Committee for case definition of MERS-CoV based on experiences of antecedent MERS-CoV outbreak in the Eastern Region of Saudi Arabia. These criteria were based on observed common signs and symptoms in order to facilitate hospital and clinic case triaging.

Diagnostic testing for MERS-CoV of the probable cases utilized duplicate naso-oropharyngeal swabs sent to the Jeddah Regional Laboratory. At the King Fahad Hospital, Jeddah, a turn-around time of less than six hours for the return of the result of PCR diagnostic testing was targeted and regularly achieved.

Sources of patient data consisted of patient chart review, Infection Control Department records, and Public Health Department records of all MERS-CoV cases at KFHJ.

Table 1. Saudi Ministry of Health criteria for suspect, probable and confirmed MERS-CoV cases (17)

\section{Suspect case (patients who should be tested for MERS-CoV)}

I. A person with fever and community-acquired pneumonia or acute respiratory distress syndrome based on clinical or radiological evidence

II. A hospitalized patient with healthcare associated pneumonia based on clinical and radiological evidence

III. A person with 1) acute febrile $\left(\geq 38^{\circ} \mathrm{C}\right)$ illness, 2$)$ body aches, headache, diarrhea, or nausea/vomiting, with or without respiratory symptoms, 3) unexplained leucopenia (WBC $<3.5 \times 10^{\circ} / \mathrm{L}$ ) and thrombocytopenia (platelets $<150 \times 10^{\circ} / \mathrm{L}$ )

IV. A person (including health care workers) who had protected or unprotected exposure to a confirmed or probable case of MERS-CoV infection and who presents with upper or lower respiratory illness within 2 weeks after exposure

\section{Probable case}

A probable case is a patient in the above category I or II with absent or inconclusive laboratory results for MERS-CoV and other possible pathogens who is a close contact of a laboratory-confirmed MERS-CoV case or who works in a hospital where MERS-CoV cases are cared for

\section{Confirmed case}

A confirmed case is a suspect case with laboratory confirmation of MERS-CoV infection 
Patients' data included biographical information (gender, nationality and age) and medical information, such as chronic co-morbidities, plan of management and whether the patient received ECMO. The data was analyzed using IBM advanced SPSS statistical package version 20 (SPSS Inc., Chicago, IL). The $\chi^{2}$ test (Fisher Exact test) was used and data were evaluated at a $5 \%$ level of significance.

\section{RESULTS}

The total sample consisted of 52 laboratory-confirmed MERS$\mathrm{CoV}$ infection patients, all of whom were admitted to the ICU of KFHJ. $40(76.9 \%)$ were male. The age of most patient was between 35 to 55 years $(46.2 \%) .9$ patients were on ECMO at least once during their ICU stay (Table 2). 39 patients died, with a $75 \%$ case-fatality rate (Fig. 1 ). Many patients had underlying comorbid medical conditions, including diabetes mellitus (51.9\%), hypertension $(46.2 \%)$, and chronic renal disease $(21.2 \%)$ (Table 3). Case-fatality rate was highest in middle-aged patients, males, patients who received antiviral therapy and statistically significant with co-morbid chronic renal failure (Table 4).

\section{DISCUSSION}

In this study, it was found that among 52 patients admitted to the ICU of the King Fahad Hospital, Jeddah, for MERS-CoV infection, most had underlying co-morbidity; $51.9 \%$ had diabetes mellitus, $46.2 \%$ had hypertension and $21.2 \%$ had chronic renal disease. In July 2013 a descriptive study by Assiri et al.

Table 2. Patient characteristics $(N=52)$

\begin{tabular}{|c|c|c|}
\hline Patient Characteristics & $\mathrm{n}$ & $\%$ \\
\hline \multicolumn{3}{|l|}{ Age } \\
\hline $15-35$ & 9 & 17.2 \\
\hline $35-55$ & 24 & 46.2 \\
\hline $55-75$ & 16 & 30.8 \\
\hline $75-85$ & 3 & 5.8 \\
\hline \multicolumn{3}{|l|}{ Gender } \\
\hline Male & 40 & 76.9 \\
\hline Female & 12 & 23.1 \\
\hline \multicolumn{3}{|l|}{ Nationality } \\
\hline Saudi & 28 & 53.8 \\
\hline Non-Saudi & 24 & 46.2 \\
\hline \multicolumn{3}{|l|}{ Patient outcome } \\
\hline Deceased & 39 & 75.0 \\
\hline Discharged & 13 & 25.0 \\
\hline \multicolumn{3}{|l|}{ Antiviral therapy } \\
\hline Received therapy & 35 & 67.3 \\
\hline Did not receive therapy & 17 & 32.7 \\
\hline \multicolumn{3}{|c|}{ Extracorporeal membrane oxygenation (ECMO) } \\
\hline Received ECMO & 9 & 17.3 \\
\hline Did not receive ECMO & 43 & 82.7 \\
\hline
\end{tabular}

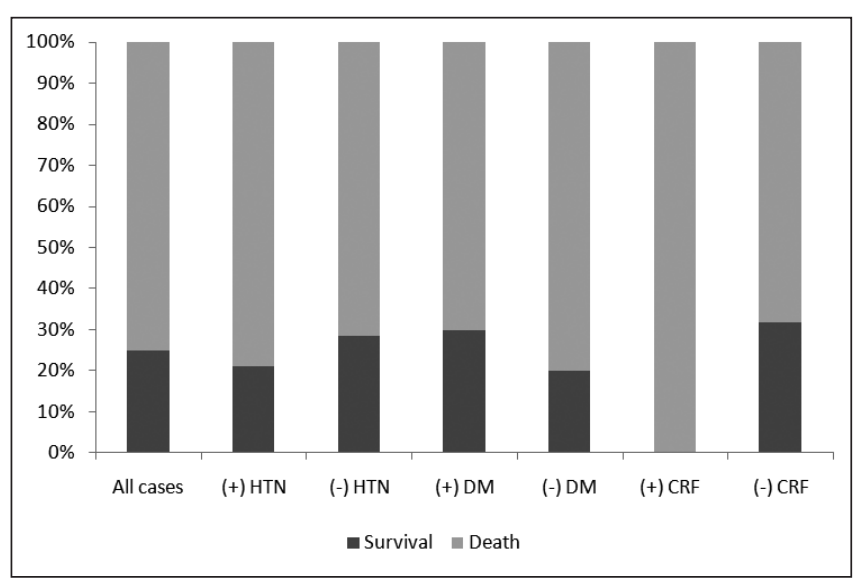

Fig. 1. Patient outcomes and correlation by co-morbidity.

HTN - hypertension, DM - diabetes mellitus, CRF - chronic renal faliure

(3), focused on the population of the Eastern provinces of Saudi Arabia over the period from September 2012 to July 2013, the corresponding co-morbidities were $68 \%$ with diabetes mellitus, $46.2 \%$ with hypertension and $49 \%$ with chronic renal disease. It is notable that in the study by Assiri et al. 23 of 47 patients were from a MERS-CoV outbreak in a renal disease unit of the Al-Ahsa hospital; this may account for the doubling of chronic renal disease in their study compared to this study.

Patients with laboratory-confirmed MERS-CoV tend to develop multi-organ failure or dysfunction, particularly severe acute respiratory disease, leading to high mortality rates. Ineffective in-vivo therapies, delays in diagnosis of MERS-CoV, and comorbidity burdens may additionally contribute to the high case fatality rate. The MERS-CoV case fatality rate has risen from initial $30 \%$ to a current rate of $41 \%$ (out of reported 781 cases 323 died, as of November 22, 2014) (1). Worldwide, the Kingdom of Saudi Arabia (KSA) has faced the highest burden of MERS$\mathrm{CoV}$ cases and case fatalities. 781 out of 904 confirmed cases of MERS-CoV (86\%) were diagnosed in KSA; 323 deaths attributed to MERS-CoV out of 347 (93\%) occurred in KSA. In contrast, the second highest global case burden and case-fatalities $(7.4 \%$ and $1.7 \%$, respectively) occurred in the neighbouring United Arab Emirates (UAE). The reasons for this significantly higher case burden and case-fatality in KSA may include more nosocomial transmissions, a population 14 times higher than UAE population, and possibly imported camel herd stocks 7 times as large as in UAE. This study demonstrated an overall mortality rate of $75 \%$

Table 3. Patient co-morbidities $(N=52)$

\begin{tabular}{|l|c|c|}
\hline Patient Co-morbidities & $\mathbf{n}$ & $\%$ \\
\hline Hypertension \\
\hline Hypertensive & 24 & 46.2 \\
\hline Non-hypertensive & 28 & 53.8 \\
\hline Diabetes mellitus \\
\hline Diabetic & 27 & 51.9 \\
\hline Non-diabetic & 25 & 48.1 \\
\hline Chronic renal failure & 11 & 21.2 \\
\hline Yes & 41 & 78.8 \\
\hline No &
\end{tabular}


Table 4. Relation between patient characteristics and co-morbidities and patient outcome $(N=52)$

\begin{tabular}{|c|c|c|c|c|c|c|}
\hline & \multicolumn{4}{|c|}{ Patient outcome } & \multirow{3}{*}{ Test } & \multirow{3}{*}{$\mathrm{p}$-value } \\
\hline & \multicolumn{2}{|c|}{ Deceased } & \multicolumn{2}{|c|}{ Discharged } & & \\
\hline & $\mathrm{n}$ & $(\%)$ & $\mathrm{n}$ & $(\%)$ & & \\
\hline \multicolumn{7}{|l|}{ Age } \\
\hline $15-35$ & 5 & 9.6 & 4 & 7.7 & \multirow{4}{*}{ Chi-square } & \multirow{4}{*}{0.082} \\
\hline $35-55$ & 16 & 30.8 & 8 & 15.4 & & \\
\hline $55-75$ & 15 & 28.8 & 1 & 1.9 & & \\
\hline $75-85$ & 3 & 5.8 & 0 & 0 & & \\
\hline \multicolumn{7}{|l|}{ Gender } \\
\hline Male & 32 & 61.5 & 8 & 15.4 & \multirow{2}{*}{ Chi-square } & \multirow{2}{*}{0.147} \\
\hline Female & 7 & 13.5 & 5 & 9.6 & & \\
\hline \multicolumn{7}{|l|}{ Nationality } \\
\hline Saudi & 22 & 42.3 & 6 & 11.5 & \multirow{2}{*}{ Chi-square } & \multirow{2}{*}{0.521} \\
\hline Non-Saudi & 17 & 32.7 & 7 & 13.5 & & \\
\hline \multicolumn{7}{|l|}{ Antiviral therapy } \\
\hline Received therapy & 28 & 53.8 & 7 & 13.5 & \multirow{2}{*}{ Chi-square } & \multirow{2}{*}{0.232} \\
\hline Did not receive therapy & 11 & 21.2 & 6 & 11.5 & & \\
\hline \multicolumn{7}{|c|}{ Extracorporeal membrane oxygenation (ECMO) } \\
\hline Received ECMO & 7 & 13.5 & 2 & 3.8 & \multirow{2}{*}{ Fisher's exact } & \multirow{2}{*}{1.000} \\
\hline Did not receive ECMO & 32 & 61.5 & 11 & 21.2 & & \\
\hline \multicolumn{7}{|l|}{ Hypertension } \\
\hline Hypertensive & 19 & 36.5 & 5 & 9.6 & \multirow{2}{*}{ Chi-square } & \multirow{2}{*}{0.749} \\
\hline Non-hypertensive & 20 & 38.5 & 8 & 15.4 & & \\
\hline \multicolumn{7}{|l|}{ Diabetes mellitus } \\
\hline Diabetic & 19 & 36.5 & 8 & 15.4 & \multirow{2}{*}{ Chi-square } & \multirow{2}{*}{0.528} \\
\hline Non-diabetic & 20 & 38.5 & 5 & 9.6 & & \\
\hline \multicolumn{7}{|l|}{ Chronic renal failure } \\
\hline Yes & 11 & 21.2 & 0 & 0 & \multirow{2}{*}{ Fisher's exact } & \multirow{2}{*}{$0.047^{*}$} \\
\hline No & 28 & 53.8 & 13 & 25 & & \\
\hline
\end{tabular}

*Statistically significant

(39 died out of 52). These were more critical ICU patients with significant disease burden of co-morbid hypertension, diabetes, chronic renal failure, and with approximately $20 \%$ receiving ECMO therapy. This was comparable to the Assiri et al. study, with a high co-morbidities burden which showed a $60 \%$ case fatality rate (28 died of 47 patients) (3).

Multiple MERS-CoV in-vitro studies have shown the benefit of broad spectrum antiviral therapies (18), the augmented effect of ribavirin to interferon (19), and the early administration of the combination antiviral therapy (13). In our study, 35 of 52 patients $(67 \%)$ received a trial of combination antiviral therapy, consisting of ribavirin in combination with interferon alfa. However, among the group receiving the antiviral therapy, 28 patients $(80 \%)$ of this cohort died. This was consistent with a small retrospective study by Al-Tawfiq et al. in which none of five patients receiving combination antiviral therapy responded and all succumbed to MERS-CoV (14). Differences may exist due to their limited sample size, as well as the late administration of antiviral therapy in the disease process - an average of 19 days after admission. For both studies, delayed diagnosis and thus delayed initiation of combination antiviral therapy likely played a part in the lack of efficacy of the antiviral therapies.

In conclusion, MERS-CoV ICU mortality remains markedly high due to a combination of factors. Current available antiviral therapies have shown a lack of response. The disease process of MERS-CoV leads to multiple organ failure, particularly respiratory and renal failure, leading to poorer outcomes.

\section{CONCLUSIONS}

Strict implementation of proper infection control practices, such as hand hygiene, personal protective equipment use, teaching of cough etiquette, room ventilation, environmental disinfection, along with continuous and close monitoring of healthcare workers compliance with infection control guidelines, are crucial to prevent the transmission of MERS-CoV infection.

To reduce mortality rates, more studies are required for better evaluation of the effect of co-morbid diseases and the mortality 
rate of MERS-CoV infection among ICU and non ICU MERS$\mathrm{CoV}$ patients.

More attention should be paid to patients with chronic renal failure attending dialysis unit due to their higher risks to contract MERS-CoV infection and hence higher mortality rates.

\section{Conflict of Interests}

None declared

\section{Adherence to Ethical Standards}

The study was approved by the Committee of Bio-Medical Ethics of Umm Al-Qura University. Informed consent was obtained from all participants included in the study.

\section{REFERENCES}

1. MERS Corona Map [Internet]. Riyadh: Saudi Ministry of Health [updated 2014 Nov 22; cited 2016 Nov 25]. Available from: http://coronamap.com/.

2. Bialek SR, Allen D, Alvarado-Ramy F, Arthur R, Balajee A, Bell D, et al.; Centers for Disease Control and Prevention (CDC). First confirmed cases of Middle East respiratory syndrome coronavirus (MERS-CoV) infection in the United States, updated information on the epidemiology of MERS-CoV infection, and guidance for the public, clinicians, and public health authorities - May 2014. MMWR Morb Mortal Wkly Rep. 2014 May 16;63(19):431-6.

3. Assiri A, Al-Tawfiq JA, Al-Rabeeah AA, Al-Rabiah FA, Al Hajjar S, Al-Barrak A, et al. Epidemiological, demographic, and clinical characteristics of 47 cases of Middle East respiratory syndrome coronavirus disease from Saudi Arabia: a descriptive study. Lancet Infect Dis. 2013 Sep;13(9):752-61.

4. World Health Organization. Interim guidance - clinical management of severe acute respiratory infections when novel coronavirus is suspected: What to do and what not to do [Internet]. [updated 2013 Feb 11; cited 2017 Nov 25]. Available from: http://www.who.int/csr/ disease/coronavirus_infections/InterimGuidance_ClinicalManagement_NovelCoronavirus_11Feb13u.pdf.

5. Centers for Disease Control and Prevention. Middle East Respiratory Syndrome (MERS). Frequently asked questions and answers [Internet]. Atlanta: CDC [updated 2014 Jul; cited 2017 Nov 25]. Available from: http://www.cdc.gov/coronavirus/mers/faq.html.

6. Centers for Disease Control and Prevention. CDC Newsroom. CDC announces first case of Middle East Respiratory Syndrome Coronavirus infection (MERS) in the United States [Internet]. Atlanta: CDC [updated 2014 May; cited 2017 Nov 25]. Available from: http://www.cdc.gov/ media/releases/2014/p0502-US-MERS.html.

7. World Health Organization. Revised interim case definition for reporting to WHO - Middle East respiratory syndrome coronavirus (MERS-CoV) [Internet]. [updated 2013 Jul 3; cited 2017 Nov 25]. Available from: http:// www.who.int/entity/csr/disease/coronavirus_infections/case_definition/ en/index.html.
8. The WHO MERS-CoV Research Group. State of knowledge and data gaps of Middle East respiratory syndrome coronavirus (MERS-CoV) in humans. PLoS Curr. 2013 Nov 12;5. pii: ecurrents.outbreaks.0bf719e35 2e7478f8ad85fa30127ddb8.

9. World Health Organization. Laboratory testing for Middle East respiratory syndrome coronavirus: interim recommendations [Internet]. [updated 2013 Sep; cited 2017 Nov 25]. Available from: http://www.who.int/entity/ csr/disease/coronavirus_infections/MERS_Lab_recos_16_Sept_2013. pdf.

10. Centers for Disease Control and Prevention. Middle East Respiratory Syndrome (MERS). Interim guidelines for collecting, handling, and testing clinical specimens from Patients Under Investigation (PUIs) for Middle East Respiratory Syndrome Coronavirus (MERS-CoV) - Version 2 [Internet]. Atlanta: CDC [updated 2013 Jun 7; cited 2017 Nov 25]. Available from: http://www.cdc.gov/coronavirus/mers/guidelinesclinical-specimens.html.

11. Corman VM, Eckerle I, Bleicker T, Zaki A, Landt O, Eschbach-Bludau $\mathrm{M}$, et al. Detection of a novel human coronavirus by real-time reversetranscription polymerase chain reaction. Euro Surveill. 2012;17(39). pii: 20285.

12. Kindler E, Jonsdottir HR, Muth D, Hamming OJ, Hartmann R, Rodriguez $\mathrm{R}$, et al. Efficient replication of the novel human betacoronavirus EMC on primary human epithelium highlights its zoonotic potential. MBio. 2013 Feb 19;(1):e00611-12.

13. Falzarano D, de Wit E, Rasmussen AL, Feldmann F, Okumura A Scott DP, et al. Treatment with interferon- $\alpha 2 \mathrm{~b}$ and ribavirin improves outcome in MERS-CoV-infected rhesus macaques. Nat Med. 2013 Sep 8;19(10):1313-7.

14. Al-Tawfiq JA, Momattin H, Dib J, Memish ZA. Ribavirin and interferon therapy in patients infected with the Middle East respiratory syndrome coronavirus: an observational study. Int J Infect Dis. 2014 Mar;20:42-6.

15. Ren Z, Yan L, Zhang N, Guo Y, Yang C, Luo Z, et al. The newly emerged SARS-like coronavirus HCoV-EMC also has an "Achilles' heel": current effective inhibitor targeting a 3C-like protease. Protein Cell. 2013 April;4(4):248-50.

16. Van Meurs K, Lally KP, Peek G, Zwischenberger JB, editors. ECMO: extracorporeal cardiopulmonary support in critical care. 3rd ed. Ann Arbor: Extracorporeal Life Support Organization; 2005.

17. Saudi Ministry of Health. Case definition and surveillance guidance for MERS-CoV testing in Saudi Arabia [Internet]. [updated 2014 May 13; cited 2017 Nov 25]. Available from: http://www.moh.gov.sa/en/CCC/ StaffRegulations/Corona/Pages/StaffRegulations.aspx.

18. Chan JF, Chan KH, Kao RY, To KK, Zheng BJ, Li CP, et al. Broadspectrum antivirals for the emerging Middle East respiratory syndrome coronavirus. J Infect. 2013 Dec;67(6):606-16.

19. Falzarano D, de Wit E, Martellaro C, Callison J, Munster VJ, Feldmann $\mathrm{H}$. Inhibition of novel $\mathrm{b}$ coronavirus replication by a combination of interferon a2b and ribavirin. Sci Rep. 2013;3:1686. doi: 10.1038/srep01686. 